False-Positive Diagnosis of Metastasis on Positron Emission Tomography-Computed Tomography Imaging Due to Hibernoma

A 45-year-old woman presented to her general practitioner with a 2-month history of a rapidly enlarging, painless mass on her left lateral upper arm. No significant past medical history was noted, and she had no personal or family history of soft tissue tumors. On clinical examination, a bilobed mass was palpable proximally within the bulk of the deltoid muscle and subcutaneous fat, with an ovoid mass extending distally. No skin involvement was apparent, and full power and range of movement were present at the shoulder. No other lesions were identified on systemic examination. Subsequent magnetic resonance imaging demonstrated an apparently malignant lesion related to the superficial aspect of the left deltoid muscle, with mixed T1 and T2 signal strength. The lesion was centered on skeletal muscle and bulged into the overlying fascia and subcutaneous adipose tissue. No bone involvement was seen. The radiologic diagnosis offered was malignant fibrous histiocytoma. Computed tomography (CT) chest scan was normal. Core biopsy of the lesion demonstrated spindle cell sarcoma of unspecified histologic subtype. Before resection, a staging positron emission tomography (PET) -CT using $\left[{ }^{18} \mathrm{~F}\right]$ fluorodeoxyglucose (FDG) was performed, which identified a moderately FDGavid lesion in the deltoid region (Fig 1, right arrow) and a second glucose-avid lesion in the subcutaneous tissues overlying the right lateral hip (Figs 1 [left arrow], 2, and 3). Core biopsy of this lesion demonstrated hibernoma. The patient proceeded to complete excision of both lesions. The deltoid lesion proved to be pleomorphic liposarcoma (Fig 4), characterized by adipogenic areas containing pleomorphic lipoblasts, intersected by fibrous septa containing atypical spindled cells with irregular nuclei, finely clumped chro-

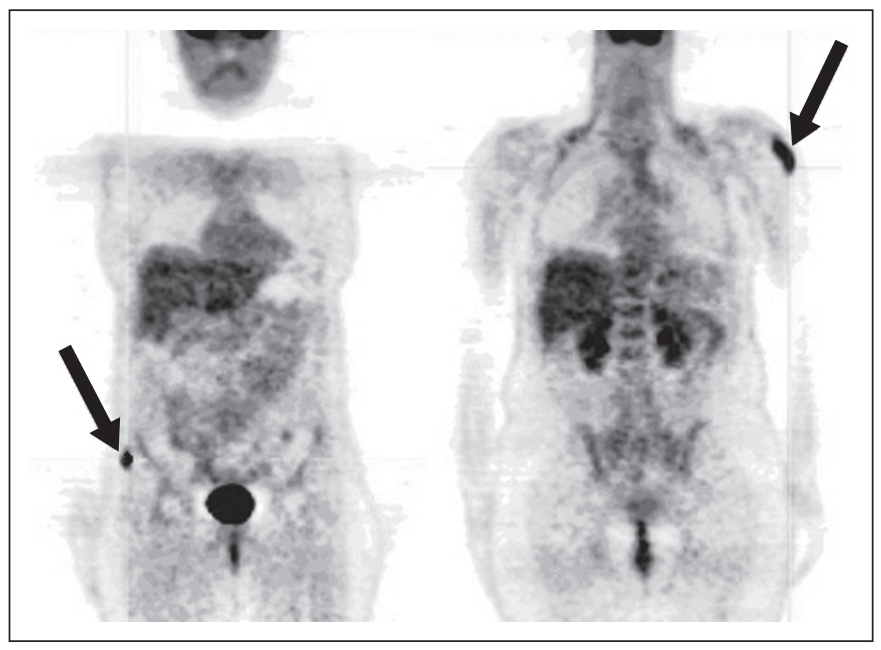

Fig 1.

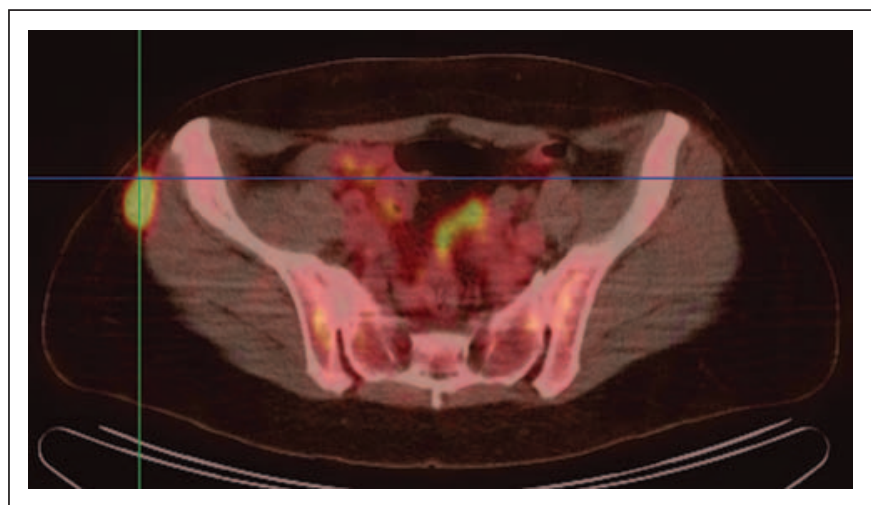

Fig 2.

matin, and one to three nucleoli. The right hip lesion was confirmed as hibernoma, characterized by a combination of mature white fat cells, multivacuolated brown fat cells, and granular eosinophilic cells (Fig 5). The patient has subsequently undergone radiotherapy to the left upper arm and follow-up scans at 3 months are clear.

Hibernoma is a rare benign tumor resembling brown fat. Brown fat is abundant in hibernating animals, and is found in the axillae and subpleural region of infants until approximately 8 weeks of age. Foci of brown fat may be found in the mediastinum, retroperitoneum, and axillae of some normal adults. Hibernoma usually arises in young adults in regions of remnant brown fat deposits, with reported cases in the scapular region and thigh, and rarely in the posterior cervical region, gluteal region, or popliteal cavity. ${ }^{1,2}$ Metastasis has never been documented. ${ }^{1}$ Magnetic resonance imaging cannot definitively differentiate hibernoma from other lipomatous lesions such as lipoma or liposarcoma, and pathological examination is vital in the diagnosis. ${ }^{3}$ Unlike other adipogenic tumors, hibernoma contains abundant mitochondria and are highly metabolically active, and therefore (unlike conventional lipoma) "hot" or glucose-avid, on PET. Glucose-avid hibernoma are described in the mediastinum, thigh, and back; ${ }^{4-6}$ this characteristic has the potential to lead to an erroneous radiologic

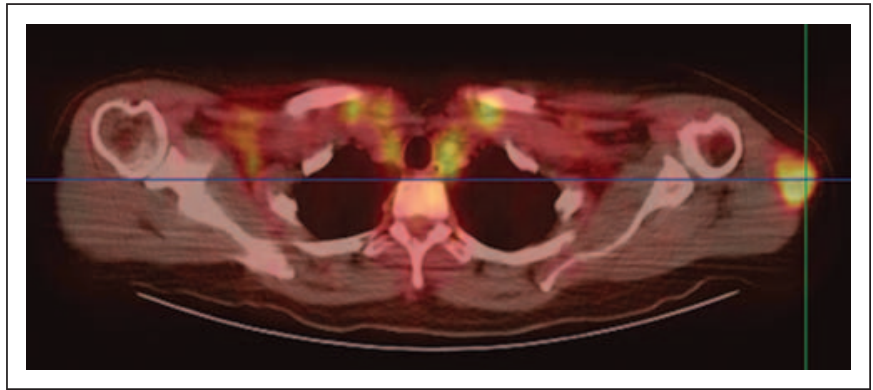

Fig 3. 


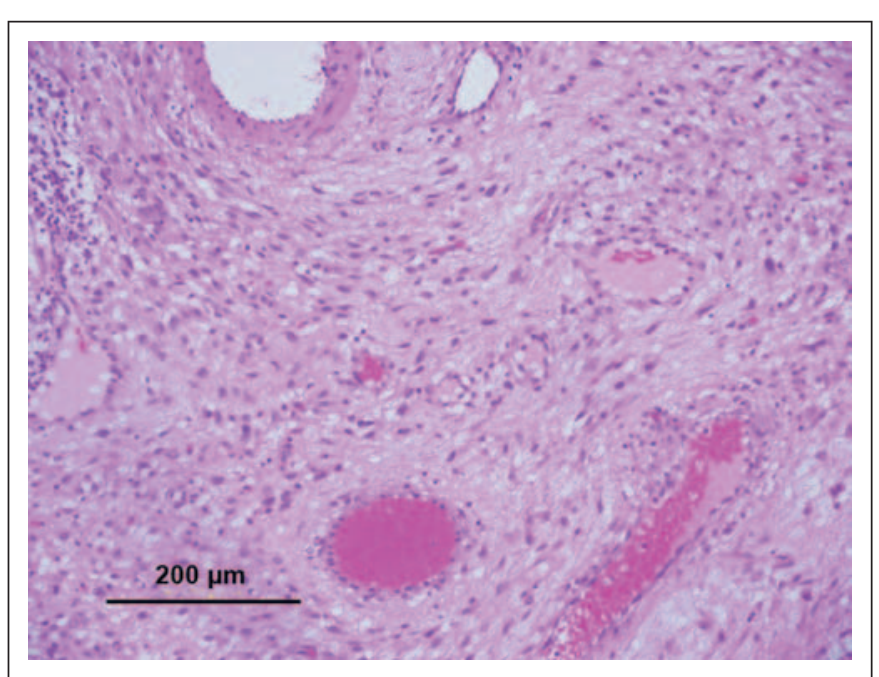

Fig 4.

diagnosis of malignancy. Hibernomas have rarely been described occurring simultaneously with another malignant tumor. One case report ${ }^{4}$ described a 40-year-old woman with a melanoma of the left thigh, who subsequently developed an intensely glucose-avid lesion in the chest wall, which was initially thought to be a metastasis. However, fine-needle aspiration and subsequent core biopsy confirmed hibernoma. A second report ${ }^{6}$ described a glucose-avid intramuscular hibernoma of the lateral chest wall found in a patient 6 months postlobectomy for lung adenocarcinoma. Another report ${ }^{7}$

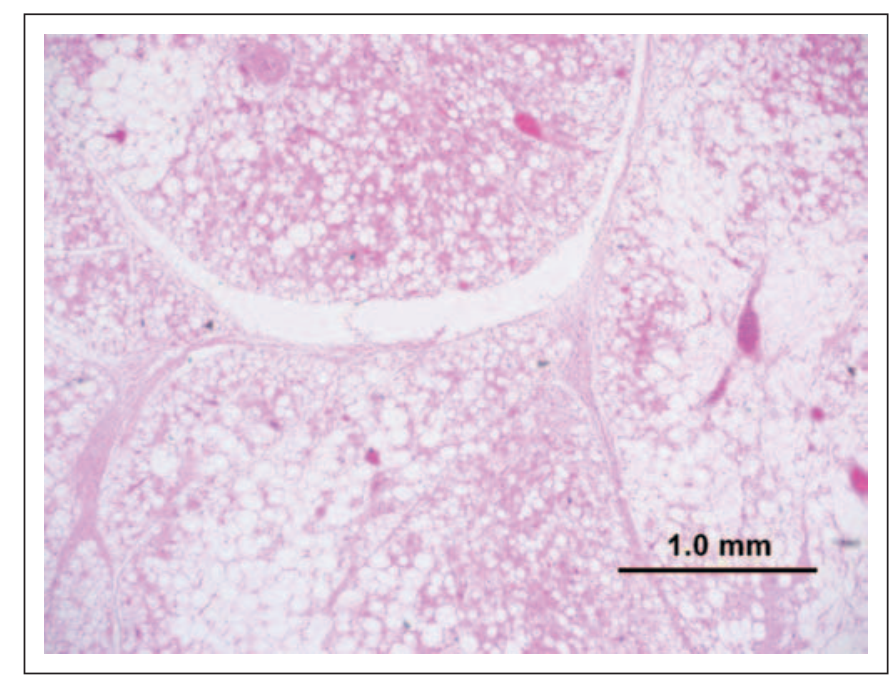

Fig 5. described a glucose-avid hibernoma in the neck found during staging of infiltrating ductal carcinoma of breast. Synchronous presentation of two different rare adipocytic tumors in our patient is perplexing and proved a diagnostic challenge. Although clinically the hip mass resembled lipoma, the marked PET-avidity in a patient with a known sarcoma elsewhere raised the spectre of metastatic disease. Hibernoma would not normally be subject to PET-CT scanning, but in the context of a known malignancy, can lead to a false-positive radiological diagnosis of metastasis. This diagnosis should be considered in PET-avid masses in the soft tissues.

\section{Sean Robison}

Australian National University Medical School, Canberra, Australian Capital Territory, Australia

\section{Andrea Rapmund}

ACT Pathology, The Canberra Hospital, Garran, Australian Capital Territory, Australia

\section{Chris Hemmings}

ACT Pathology, The Canberra Hospital, Garran; Canberra Sarcoma Group, c/o Calvary Clinic, Bruce, Australian Capital Territory, Australia

\section{Michael Fulham}

Department of PET and Nuclear Imaging, Royal Prince Alfred Hospital, Camperdown; Faculty of Medicine and School of Information Technologies, University of Sydney, Sydney, New South Wales, Australia

\section{Peter Barry}

Canberra Sarcoma Group, c/o Calvary Clinic, Bruce, Australian Capital Territory, Australia

\section{AUTHORS' DISCLOSURES OF POTENTIAL CONFLICTS OF INTEREST}

The author(s) indicated no potential conflicts of interest.

\section{REFERENCES}

1. Furlong MA, Fanburg-Smith JC, Miettinen M: The morphologic spectrum of hibernoma: A clinicopathologic study of 170 cases. Am J Surg Pathol 25:809-814, 2001

2. Chen DY, Wang CM, Chan HL: Hibernoma: Case report and literature review. Dermatol Surg 24:393-395, 1998

3. Ritchie DA, Aniq H, Davies AM, et al: Hibernoma: correlation of histopathology and magnetic-resonance-imaging features in 10 cases. Skeletal Radiol 35:579-589, 2006

4. Subramaniam RM, Clayton AC, Karantanis D, et al: Hibernoma: F-18 FDG PET/CT imaging. J Thorac Oncol 2:569-570, 2007

5. Tsuchiya T, Osanai T, Ishikawa A, et al: Hibernomas show intense accumulation of FDG positron emission tomography. J Comput Assist Tomogr 30:333336, 2006

6. Tsunezuka $Y$, Kobayashi $T$, Katayanagi $K$, et al: Intramuscular tumor detected by FDG positron emission tomography scanning following postoperative lung cancer: Hibernoma of the right back. Respiration 74:341-343, 2007

7. Giblin E, Lynn D, Mortman K: Cervical hibernoma demonstrating uptake on Tc-99m lymphoscintigraphy. Clin Nucl Med 31:694-696, 2006

DOI: 10.1200/JCO.2008.19.4977; published online ahead of print at www.jco.org on December 29, 2008 